04

\title{
Минимизация суммарной глубины внутренних пилообразных рельефов двухслойной рельефно-фазовой дифракционной микроструктуры
}

\author{
(С) Г.И. Грейсух ${ }^{1}$, В.А. Данилов ${ }^{2}$, С.А. Степанов ${ }^{1}$, А.И. Антонов ${ }^{1}$, Б.А. Усиевич ${ }^{3}$ \\ ${ }^{1}$ Пензенский государственный университет архитектуры и строительства, \\ 440028 Пенза, Россия \\ ${ }^{2}$ Научно-технологический центр уникального приборостроения РАН, \\ 117342 Москва, Россия \\ ${ }^{3}$ Институт общей фоизики им. А.М. Прохорова РАН, \\ 119991 Москва, Россия \\ e-mail: grey@pguas.ru
}

Поступила в редакцию 15.06.2017 г.

В окончательной редакции 08.08.2017 г.

\begin{abstract}
Представлены результаты исследования возможности уменьшения суммарной глубины рельефов двухслойной микроструктуры, имеющей два внутренних пилообразных микрорельефа, обеспечивающих снижение зависимости дифракционной эффективности микроструктуры от длины волны излучения и угла падения излучения на микроструктуру. Эти результаты позволяют минимизировать трудоемкость получения в рамках электромагнитной теории дифракции оптимальных глубин микрорельефов в зависимости от требований, предъявляемых к дифракционному оптическому элементу. Оптимальные глубины обеспечат в заданных спектральном диапазоне и интервале углов падения излучения максимально возможное (для выбранной ширины самой узкой зоны пилообразного микрорельефа) значение дифракционной эффективности в точке ее минимума.
\end{abstract}

DOI: 10.21883/OS.2018.01.45365.143-17

\section{1. Введение}

Одним из факторов, ограничивающих широкое использование дифракционных оптических элементов (ДОЭ) в изображающих оптических системах, рассчитанных на полихроматическое излучение, по-прежнему остается зависимость их дифракционной эффективности (ДЭ) от длины волны и угла падения излучения на микроструктуру. Например, в случае простейшей однослойной пилообразной рельефно-фазовой микроструктуры (РФМ) ДЭ в первом рабочем порядке, близкой к единице, может быть получена только на одной длине волны и при одном значении угла падения излучения на микроструктуру. На других длинах волн и при других углах падения излучения ДЭ в рабочем порядке падает, a в побочных порядках растет, что может привести к возникновению цветного ореола (гало), сопровождающего наиболее яркие фрагменты изображения, формируемого оптической системой в полихроматическом излучении.

Интегрально уровень побочных дифракционных порядков, как правило, оценивается по отличию ДЭ в первом рабочем порядке от единицы. Известный метод снижения зависимости ДЭ РФМ от длины волны и угла падения излучения на микроструктуру предполагает ее компоновку, как минимум, из пары оптических материалов. Причем в случае однорельефной микроструктуры, представленной на рис. 1, значения коэффициента дисперсии $v$ и показателя преломления $n$ выбранной пары материалов должны удовлетворять условию: если $v_{1}>v_{2}$, то и $n_{1}>n_{2}$. То есть у кроноподобного оптического материала показатель преломления должен быть больше, чем у флинтоподобного [1]. Данное условие сегодня не позволяет использовать для компоновки однорельефных РФМ пар оптических пластмасс наиболее технологичных и коммерчески доступных марок.

Указанное ограничение снимается с переходом к двухрельефным РФМ, представленным на рис. 2, $a, b$ [2-5].

Обе эти микроструктуры с точки зрения скалярной теории дифракции (СТД) идентичны, так как считаются бесконечно тонкими транспарантами, каждый из которых вносит в нормально падающий на него плоский волновой фронт определенную фазовую задержку. Эти

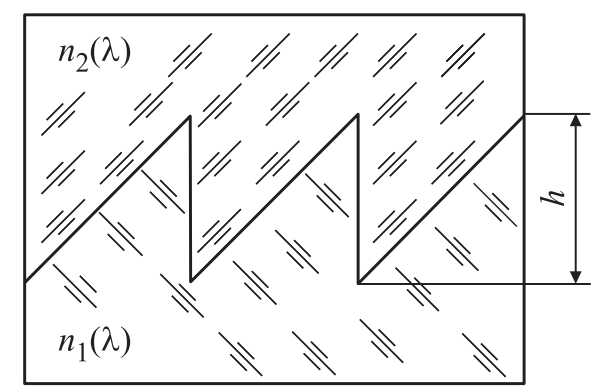

Рис. 1. Двухслойная однорельефная пилообразная микроструктура. 
фазовые задержки и соответственно ДЭ в рамках СТД при равенстве одинаково обозначенных показателей преломления и глубин совпадают [2]. При переходе к наклонному падению излучения на ДОЭ концепция бесконечно тонкого транспаранта используется также (см., например, работы [3-6]). Ее адекватность независимо от компоновки, числа слоев и рельефов экспериментально подтверждена в работе [7]. В результате в рамках СТД ДЭ РФМ в первом рабочем порядке дифракции рассчитывается по формуле $[3,4,6]$

$$
\eta=\left\{\frac{\sin (\pi-0.5 \Delta \varphi)}{\pi-0.5 \Delta \varphi}\right\}^{2}
$$

При этом микроструктуры, представленные на рис. $2, a, b$, вносят в падающий на них волновой фронт фазовую задержку $\Delta \varphi$, определяемую одним и тем же уравнением

$$
\begin{array}{r}
\Delta \varphi=\frac{2 \pi}{\lambda}\left[h_{1}\left(\sqrt{n_{1}^{2}(\lambda)-\sin ^{2} \theta}-\cos \theta\right)\right. \\
\left.-h_{2}\left(\sqrt{n_{2}^{2}(\lambda)-\sin ^{2} \theta}-\cos \theta\right)\right],
\end{array}
$$

где $\lambda$ - длина волны падающего на микроструктуру излучения, $h_{i}$ и $n_{i}(\lambda)$ - как это показано на рис. 2, соответственно глубины рельефов и показатели преломления материалов, из которых эти рельефы выполнены, $\theta-$ угол падения излучения на ДОЭ из воздуха в среду с показателем преломления $n_{1}(\lambda)$.

С точки зрения массового производства микроструктуры, представленные на рис. 2, $a, b$, далеко не идентичны: микроструктура с двумя внутренними рельефами более технологична по сравнению с микроструктурой с внутренним и наружным рельефами.

Оценки ДЭ РФМ, полученные по формулам (1) и (2), сопоставлялись с результатами, полученными в рамках строгой теории дифракции, основанной на решении системы уравнений Максвелла с использованием строгого метода связанных волн (Rigorous coupled-wave analysis (RCWA)). В соответствии с ним пилообразные рельефы дифракционной микроструктуры заменялись ступенчатыми, и каждая ступень ограничивала тонкий слой, образующий бинарную диэлектрическую решетку, к которой применялось разложение в ряд Фурье [8]. Наши исследования, выполненные RCWA-методом с использованием компьютерной программы, представленной в [9], показали, что в случае двухслойной микроструктуры с внутренним и наружным рельефами (рис. 2,a) хорошая сходимость результатов расчета, а следовательно, и их высокая достоверность достигается при высоте ступеней порядка $0.2 \lambda_{\min }$ и числе гармоник ряда Фурье большем 300 [4]. Здесь $\lambda_{\min }$ - минимальная длина волны рабочего спектрального диапазона. Такие же ограничения на высоту ступеней и число гармоник обеспечивают хорошую сходимость результатов расчета
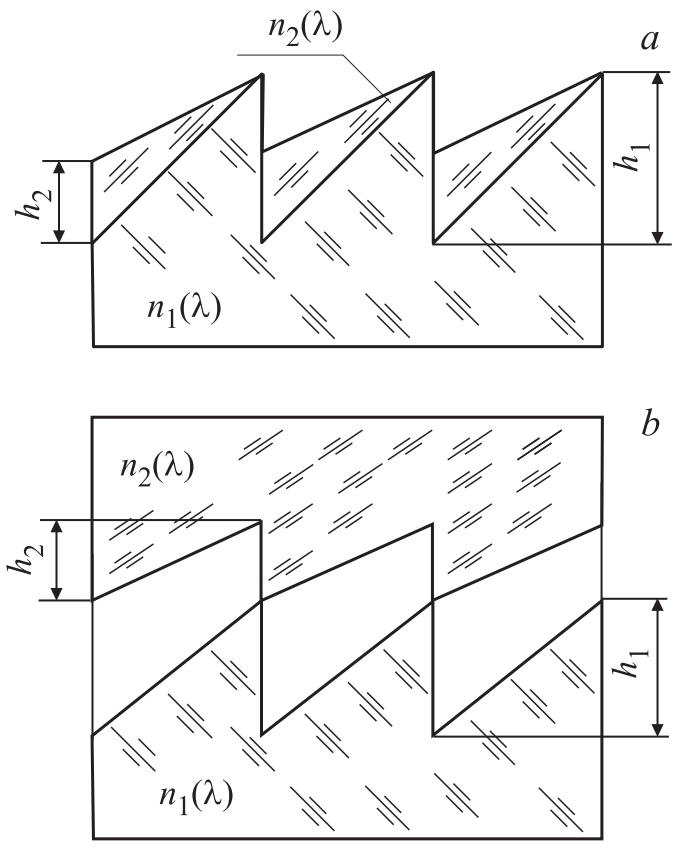

Рис. 2. Двухслойные двухрельефные пилообразные микроструктуры: $a-$ с внутренним и наружным рельефами, $b-$ с двумя внутренними рельефами.

и в случае микроструктуры с двумя внутренними рельефами (рис. 2, $b$ ).

Сопоставительный анализ оценок ДЭ РФМ, полученных в рамках СТД и RCWA-методом [4,5], показал, что достоверность оценки по СТД зависит от относительного периода микроструктуры, т. е. от отношения периода микроструктуры к суммарной глубине рельефов $\Lambda / h_{\Sigma}$. При этом принципиальным является то, что оценки по СТД всегда завышены по сравнению с оценками, полученными RCWA-методом, и степень завышения тем больше, чем меньше $\Lambda / h_{\Sigma}[5]$.

Здесь следует заметить, что ДОЭ, включаемый во вращательно-симметричную оптическую систему с целью коррекции хроматизма и монохроматических аберраций, имеет кольцевую квазипериодическую микроструктуру, и за характерный пространственный период $\Lambda$ мы будем принимать ширину самой узкой зоны пилообразного микрорельефа.

Нетрудно видеть (рис. 2, $a, b)$, что у микроструктуры с внутренним и наружным микрорельефами $h_{\Sigma}=h_{1}$, а у микроструктуры с двумя внутренними микрорельефами $h_{\Sigma}=h_{1}+h_{2}$. Отсюда следует, что задача уменьшения суммарной глубины рельефов наиболее актуальна для РФМ с двумя внутренними микрорельефами. Не менее актуальна для такой РФМ и минимизация трудоемкости получения (в рамках строгой теории дифракции) оптимальных глубин двух внутренних микрорельефов в зависимости от требований, предъявляемых к ДОЭ. Решению вышеуказанных задач и посвящена настоящая работа. 
Таблица 1. Минимальная нормированная ДЭ, полученная RCWA-методом и в рамках CTД, для ряда значений углов падения излучения на двухслойную микроструктуру с внутренним и наружным рельефами

\begin{tabular}{c|c|c|c|c|c|c}
\hline \multirow{2}{*}{$\Lambda / h_{1}$} & \multicolumn{2}{|c|}{$\eta_{\mathrm{EM}, \min }^{(\psi)} / \eta_{\mathrm{EM}, \max }^{(\psi=0)}$} & \multicolumn{3}{|c}{$\eta_{\mathrm{S}, \min }^{(\theta)} / \eta_{\mathrm{S}, \max }^{(\theta=0)}$} \\
\cline { 2 - 6 } & $\psi=10^{\circ}$ & $\psi=15^{\circ}$ & $\psi=20^{\circ}$ & $\theta=10^{\circ}$ & $\theta=15^{\circ}$ & $\theta=20^{\circ}$ \\
\hline 10 & 0.933 & 0.925 & 0.873 & \multicolumn{3}{|c}{0.952} \\
20 & 0.950 & 0.950 & 0.909 & \multicolumn{3}{|c}{}
\end{tabular}

\section{Спектральная и угловая зависимости ДЭ РФМ при оптимальных глубинах рельефов, полученных RCWA-методом}

Оптимальные значения глубин рельефов $h_{1}$ и $h_{2}$ для выбранной пары оптических материалов зависят от выбора критерия оптимальности. Если предполагается использовать ДОЭ в качестве корректора аберраций изображающей оптической системы, то для исключения гало наиболее адекватным является критерий, предложенный в работе [5]. В соответствии с ним за оптимальные значения глубин $h_{1}$ и $h_{2}$ принимаются значения, обеспечивающие в выбранном спектральном диапазоне максимально возможный интервал углов падения излучения, в пределах которого ДЭ (в точке ее минимума) не опускалась ниже заданного уровня, обеспечивающего отсутствие гало.

С использованием описанного выше критерия сопоставительный анализ оценок ДЭ, полученных в рамках скалярной и строгой теорий дифракции в ходе всесторонних исследований двухслойной микроструктуры, состоящей из полиметилметакрилата и поликарбоната, проводился ранее в видимом спектральном диапазоне $0.4 \leq \lambda \leq 0.7 \mu \mathrm{m}$ только в варианте с внутренним и наружным микрорельефами [5]. Основные результаты этого анализа представлены в табл. 1, заимствованной из указанной работы для удобства сопоставления их с результатами исследования двухслойной микроструктуры с двумя внутренними рельефами. Сразу же заметим, что в этой таблице для сопоставления углов падения излучения на микроструктуру, приводящих к снижению ДЭ до одного и того же уровня, но полученных двумя различными методами, эти углы обозначены разными буквами: $\theta-$ в рамках СТД и $\psi-\mathrm{RCWA-методом.} \mathrm{Это}$ вызвано тем, что в силу асимметрии рельефа модуль отрицательного угла падения $\left|\psi_{\mathrm{N}}\right|$ и положительный угол падения излучения $\psi_{\mathrm{P}}$, приводящие к снижению ДЭ, оцениваемого RCWA-методом, до одного и того же уровня могут существенно отличаться и за оценочный угол $\psi$ и в работе [5], и в настоящей работе принят наименьший из углов $\left|\psi_{\mathrm{N}}\right|$ и $\psi_{\mathrm{P}}$ падения излучения из воздуха на ДОЭ, приводящий к снижению ДЭ до заданного уровня.
В спектральном диапазоне $0.4 \leq \lambda \leq 0.7 \mu \mathrm{m}$ оптимальные значения глубин рельефов двухслойной двухрельефной микроструктуры, состоящей из полиметилметакрилата и поликарбоната, даваемые СТД, составляют в полиметилметакрилате $h_{1}=15.1 \mu \mathrm{m}$ и в поликарбонате $h_{2}=11.7 \mu \mathrm{m}$ [5]. В таблице 1 , как и далее, $\eta_{\mathrm{EM}, \min }^{(\psi)}$ и $\eta_{\mathrm{S}, \min }^{(\theta)}-$ минимальные значения Дэ в пределах выбранного спектрального диапазона при заданном угле падения излучения на ДОЭ, полученные RCWA-методом и в рамках СТД, а $\eta_{\mathrm{EM}, \max }^{(\psi=0)}$ и $\eta_{\mathrm{S}, \max }^{(\theta=0)}-$ максимальные значения ДЭ в пределах выбранного спектрального диапазона, полученные соответствующим методом, но при нормальном падении излучения на ДОЭ $(\psi=\theta=0)$.

Сразу же отметим, что в настоящей работе, так же как и в работах $[4,5]$, при расчете ДЭ RCWA-методом (в отличие от расчета в рамках СТД) учитываются френелевские потери, обусловленные отражением излучения от обоих рельефов. Это и является первопричиной отличия максимальных (в пределах выбранного спектрального диапазона) значений ДЭ, достигаемых при $\psi=\theta=0$, т.е. $\eta_{\mathrm{EM}, \max }^{(\psi=0)}$ и $\eta_{\mathrm{S}, \max }^{(\theta=0)}$, полученных RCWA-методом и в рамках СТД соответственно.

Прежде чем перейти к представлению результатов наших исследований РФМ с двумя внутренними рельефами, отметим, что подобные исследования, но с использованием в качестве оценочной функции проинтегрированной по спектру ДЭ, уже проводились в ряде работ (см., например, $[10,11])$. Очевидно, что использование такой оценочной функции вполне уместно при анализе ДОЭ, предназначенных для концентрации полихроматического излучения, например солнечного. Однако такая оценочная функция мало чувствительна к провалам ДЭ на отдельных участках выбранного спектрального диапазона, а именно такие провалы могут приводить к возникновению гало у изображающих оптических систем.

В табл. 2 представлены полученные в рамках скалярной и строгой теорий дифракции результаты наших исследований РФМ с двумя внутренними рельефами, состоящей из полиметилметакрилата и поликарбоната. Глубина рельефа в полиметилметакрилате $h_{1}=15.1 \mu \mathrm{m}$, а глубина рельефа в поликарбонате при расчете RCWAметодом оптимизировалась и превышала полученную в рамках СТД $h_{2}=11.7 \mu \mathrm{m}$ не более чем на $0.01 \mu \mathrm{m}$.

Сопоставляя данные табл. 1 и 2, легко видеть, что переход к РФМ с двумя внутренними рельефами привел в представляющем интерес интервале углов падения излучения $\left(0 \leq \psi \leq 15^{\circ}\right)$ лишь к незначительному падению ДЭ (порядка 3-4\%), но это потребовало увеличения периода микроструктуры $\Lambda$ почти в 1.8 раза.

Исследование возможности уменьшения периода путем уменьшения суммарной глубины рельефов включало поиск оптимальных значений глубины рельефа $h_{2}$ для ряда убывающих значений глубины $h_{1}$. При этом за оптимальное принималось значение глубины рельефа $h_{2}$, которое обеспечивало в спектральном диапазоне 
Таблица 2. Минимальная нормированная ДЭ, полученная RCWA-методом и в рамках СТД, для ряда значений углов падения излучения на двухслойную микроструктуру с двумя внутренними рельефами при $h_{\Sigma}=26.81 \mu \mathrm{m}$

\begin{tabular}{c|c|c|c|c|c|c}
\hline \multirow{2}{*}{$\Lambda / h_{1}$} & \multicolumn{2}{|c|}{$\eta_{\mathrm{EM}, \min }^{(\psi)} / \eta_{\mathrm{EM}, \max }^{(\psi=0)}$} & \multicolumn{3}{|c}{$\eta_{\mathrm{S}, \min }^{(\theta)} / \eta_{\mathrm{S}, \max }^{(\theta=0)}$} \\
\cline { 2 - 6 } & $\psi=10^{\circ}$ & $\psi=15^{\circ}$ & $\psi=20^{\circ}$ & $\theta=10^{\circ}$ & $\theta=15^{\circ}$ & $\theta=20^{\circ}$ \\
\hline 10 & 0.900 & 0.897 & 0.843 & \multicolumn{3}{|c}{0.952} \\
20 & 0.942 & 0.940 & 0.892 & \multicolumn{2}{|c}{}
\end{tabular}

$0.4 \leq \lambda \leq 0.7 \mu \mathrm{m}$ и в интервале углов падения излучения $0 \leq \psi \leq 15^{\circ}$ максимально возможное значение ДЭ в точке ее минимума. Поиск показал, что в достаточно широком диапазоне значений глубины рельефа в полиметилметакрилате $8 \leq h_{1} \leq 15.1 \mu$ т и при $10 \leq \Lambda / h_{\Sigma} \leq 20$ зависимость оптимальных значений $h_{2}$ от $h_{1}$ почти строго линейная:

$$
h_{2} \approx a+b h_{1} .
$$

Здесь $a=-0.90 \mu \mathrm{m}, b=0.84$. Линейность указанной зависимости, сохраняющаяся для всех практически приемлемых пар материалов, из которых может компоноваться двухслойная РФМ, существенно облегчает поиск оптимального значения одной из глубин рельефа при заданном значении другой.

В табл. 3 представлены полученные в рамках строгой теории дифракции результаты оценки ДЭ для двух уменьшенных и характерных значений суммарной глубины рельефов.

Сопоставляя данные табл. 1-3, нетрудно видеть, что при одинаковых отношениях $\Lambda / h_{\Sigma}$ нормированные ДЭ микроструктур с одним внутренним и с двумя внутренними микрорельефами, полученные RCWA-методом, весьма близки при глубинах рельефов, определенных СТД-методом $\left(h_{1}=15.1 \mu \mathrm{m}\right.$ и $\left.h_{2}=11.7 \mu \mathrm{m}\right)$. При уменьшении суммарной глубины рельефов микроструктуры с двумя внутренними микрорельефами нормированная ДЭ монотонно снижается. В то же время характер зависимостей ДЭ от длины волны и угла падения излучения

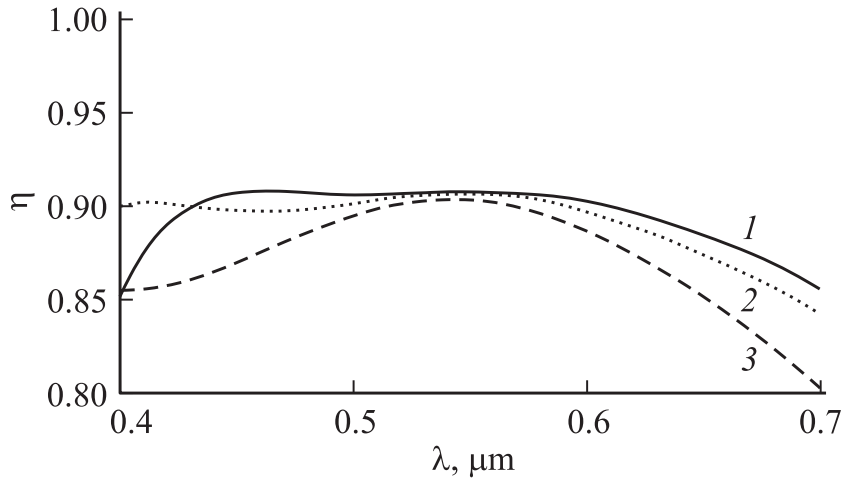

Рис. 3. Зависимости ДЭ, полученные методом RCWA при $\Lambda / h_{\Sigma}=20$, от длины волны излучения, падающего вдоль нормали к подложке микроструктуры: $1-h_{\Sigma}=26.81,2-21.12$, $3-13.78 \mu \mathrm{m}$.

сохраняется (ср., например, кривые рис. 3 с кривыми рис. 4 работы [4] или рис. 3 работы [5]).

В работе [12] показано, что отрицательное влияние побочных дифракционных порядков на качество изображения, формируемого оптической системой с ДОЭ, может не сказываться при снижении нормированной ДЭ вплоть до 0.85. Следовательно, имеется возможность значительного уменьшения $h_{\Sigma}$ и соответственно минимально допустимой ширины самой узкой зоны пилообразного микрорельефа до уровня, необходимого для эффективной коррекции хроматизма и монохроматических аберраций оптической системы, без риска снижения качества изображения из-за побочных порядков дифракции и, в частности, без риска появления гало.

\section{Заключение}

Результаты, представленные в настоящей работе, позволяют существенно снизить трудоемкость получения в рамках строгой теории дифракции оптимальных глубин двух внутренних микрорельефов двухслойной микроструктуры в зависимости от требований, предъявляемых

Таблица 3. Полученные RCWA-методом результаты оценки влияния суммарной глубины рельефов на Дэ микроструктуры с двумя внутренними рельефами

\begin{tabular}{|c|c|c|c|c|c|c|c|}
\hline$\Lambda / h_{\Sigma}$ & $\psi, \circ$ & $h_{1}, \mu \mathrm{m}$ & $h_{2}, \mu \mathrm{m}$ & $h_{\Sigma}, \mu \mathrm{m}$ & $\eta_{\mathrm{EM}, \min }^{(\psi)}$ & $\eta_{\mathrm{EM}, \text { max }}^{(\psi=0)}$ & $\eta_{\mathrm{EM}, \min }^{(\psi)} / \eta_{\mathrm{EM}, \text { max }}^{(\psi=0)}$ \\
\hline \multirow{4}{*}{10} & \multirow{2}{*}{10} & 8 & 5.78 & 13.78 & 0.763 & 0.882 & 0.865 \\
\hline & & 12 & 9.12 & 21.12 & 0.814 & 0.898 & 0.906 \\
\hline & \multirow{2}{*}{15} & 8 & 5.78 & 13.78 & 0.727 & 0.882 & 0.824 \\
\hline & & 12 & 9.12 & 21.12 & 0.796 & 0.898 & 0.887 \\
\hline \multirow{4}{*}{20} & \multirow{2}{*}{10} & 8 & 5.78 & 13.78 & 0.799 & 0.889 & 0.898 \\
\hline & & 12 & 9.12 & 21.12 & 0.837 & 0.902 & 0.929 \\
\hline & \multirow{2}{*}{15} & 8 & 5.78 & 13.78 & $0.758 ?$ & 0.889 & 0.852 \\
\hline & & 12 & 9.12 & 21.12 & 0.832 & 0.902 & 0.923 \\
\hline
\end{tabular}


к ДОЭ. Эти глубины обеспечат в заданных спектральном диапазоне и интервале углов падения излучения максимально возможное (для выбранной ширины самой узкой зоны пилообразного микрорельефа) значение ДЭ в точке ее минимума.

Несмотря на то, что представленные закономерности продемонстрированы на примере микроструктуры, скомпонованной только из одной пары коммерчески доступных оптических пластмасс - полиметилметакрилата и поликарбоната, они имеют общий характер. Действительно, замена материалов приводит лишь к изменению оптимальных значений глубин рельефов, даваемые СТД, и изменению значений размерного и безразмерного коэффициентов $a$ и $b$ в уравнении (3). К сожалению, другим парам из коммерчески доступных сегодня оптических пластмасс соответствуют большие оптимальные значения глубин рельефов, даваемые СТД. Именно этим и объясняется выбор авторами пары полиметилметакрилат/поликарбонат.

Двухслойные микроструктуры с минимизированной суммарной глубиной внутренних пилообразных рельефов представляют практический интерес и могут найти применение в изображающих системах, проекционных дисплеях и т.д.

Работа выполнена при финансовой поддержке Российского научного фонда (проект № 17-19-01461).

\section{Список литературы}

[1] Грейсух Г.И., Ежсов Е.Г., Степанов С.А. // Компьютерная оптика. 2008. Т. 32. № 1. С. 43.

[2] Грейсух Г.И., Безус Е.А., Быков Д.А., Ежов Е.Г., Степанов С.A. // Опт. и спектр. 2009. Т. 106. № 4. С. 694.

[3] Greisukh G.I., Danilov V.A., Ezhov E.G., Stepanov S.A., Usievich B.A. // Opt. Commun. 2015. V. 338. P. 54.

[4] Грейсух Г.И., Данилов В.А., Ежов Е.Г., Степанов С.А., Усиевич Б.А. // Опт. и спектр. 2015. Т. 118. № 6. С. 118.

[5] Грейсух Г.И., Данилов В.А., Ежов Е.Г., Степанов С.А., Усиевич Б.А. // Оптический журнал. 2015. Т. 82. № 5. С. 56.

[6] Zhao Y.H., Fan C.J., Ying C.F., Liu S.H. // Opt. Commun. 2013. V. 295. P. 104.

[7] Choi J., Cruz-Cabrera A.A., Tanbakuchi A. Spectral Diffraction Efficiency Characterization of Broadband Diffractive Optical Elements. Sandia report SAND20132603. New Mexico: Sandia National Laboratories, 2013. 30 p.

[8] Moharam M.G., Gaylord T.K. // J. Opt. Soc. Am. 1982. V. 72. N 10. P. 1385.

[9] Lyndin N.M. Modal and C Methods Grating Design and Analysis Software. [Электронный ресурс] Режим доступа: http://www.mcgrating.com

[10] Yang H., Xue C., Li C., Wang J. // Appl. Opt. 2016. V. 55. N 7. P. 1675.

[11] Yang H., Xue C., Li C., Wang J., Zhang R. // Appl. Opt. 2016. V. 55. N 25. P. 7126.

[12] Грейсух Г.И., Данилов В.А., Ежсов Е.Г., Степанов С.А., Усиевич Б.А. // Оптический журнал. 2016. Т. 83. № 3. С. 27. 